Conclusions Biofeedback is associated with significant improvement in the quality of life as well as possible improvements in symptoms related to both incontinence and evacuatory disorders. It is probably an underused service. Further larger prospective studies are required to assess the efficacy of biofeedback in pouch related dysfunction.

\section{IDDF2018-ABS-0059 IMMUNOGENICITY OF QUADRIVALENT INFLUENZA VACCINE FOR PATIENTS WITH INFLAMMATORY BOWEL DISEASE}

Yasuhisa Sakata*, Shimpei Shirai, Megumi Hara, Nanae Tsuruoka, Ryo Shimoda, Kazuma Fujimoto. Saga Medical School, Japan

\subsection{6/gutjnl-2018-IDDFabstracts.91}

Background No reports have described the immunogenicity and boosting effect of the quadrivalent influenza vaccine in adults-patients with inflammatory bowel disease (IBD).

Methods Adults with Crohn's disease or ulcerative colitis were randomly assigned to a single vaccination group or booster group, and a quadrivalent influenza vaccine was administered subcutaneously. Serum samples were collected before vaccination, 4 weeks after vaccination and after the influenza season in the single vaccination group and before vaccination, 4 weeks after the first vaccination, 4 weeks after the second vaccination, and after the influenza season in the booster group. We measured hemagglutination inhibition antibody titer and calculated the geometric mean titer ratio, seroprotection rate, and seroconversion rate.

Results Totally, 132 patients were enrolled. Twenty-two patients received immunomodulatory monotherapy and 16 received anti-TNF- $\alpha$ single-agent therapy. Fifteen patients received combination therapy comprising an immunosuppressant and anti-TNF- $\alpha$ agent. Each vaccine strain showed immunogenicity satisfying the European Medicines Agency criteria with a single inoculation. The booster influenza vaccination did not induce an additional response. In patients administered infliximab, the seroprotection rate and seroconversion rate tended to be lower in patients who maintained blood concentrations (seroprotection rate: H1N1: OR, $0.37 \quad(95 \%$ CI, 0.11-1.21); H3N2: 0.22 (0.07-0.68); seroconversion rate: H1N1: 0.23 (0.06-0.91); H3N2: 0.19 (0.06-0.56)).

Conclusions Although single-dose quadrivalent influenza vaccine showed sufficient immunogenicity in the patients with inflammatory bowel disease, the immunogenicity of patients receiving infliximab therapy was low.

\section{IDDF2018-ABS-0060 A STUDY ON THE STATUS OF PROTON PUMP INHIBITOR PRESCRIBED FOR HOSPITAL INPATIENTS IN JAPAN}

Yasuhisa Sakata*, Nanae Tsuruoka, Ryo Shimoda, Daisuke Yamaguchi, Kazuma Fujimoto. Saga Medical School, Japan

\subsection{6/gutjnl-2018-IDDFabstracts.92}

Background In Japan, the amount of usage of proton pump inhibitors (PPIs) has been increasing as the indications extended to maintenance treatment of reflux esophagitis and prevention of ulcer recurrence in patients receiving non-steroidal anti-inflammatory agents (NSAIDs) and low-dose aspirins, in addition to the treatment of peptic ulcer. This study aimed to clarify the status of use of PPIs in hospitalised patients with Diagnosis Procedure Combination (DPC) data.

Methods A retrospective observational study was carried out. We analysed the PPI prescription data for past 8 years (2009_ 2016) using the DPC survey data of approximately ten thousand patients a year in Saga university hospital. The primary objective is to investigate the trend of a number of hospitalised patients who were prescribed PPI for the past 8 years. The secondary objective is to investigate the changes in the characteristics of patients (age, indication, concomitant medication, and clinical department that follows a patient). Lists of proton pump inhibitors subjected to the survey are omeprazole, esomeprazole, lansoprazole, rabeprazole and vonoprazan.

Results We identified 11009 patients using PPIs throughout the study period. Prevalence of PPI use was higher among male than female, approximately 1.3 times. The average age of PPI users was 67.8 years. The number of PPI users increased 1.5 times during the study period, from 1071 (10.5\% of inpatients) in 2009 to 1581 (12.3\% of inpatients) in 2016. Lansoprazole was the most commonly used agent $(30.7 \%-50.5 \%)$ during the study period, followed by rabeprazole (29.3\%-48.6\%) and esomeprazole. A newer agent, vonoprazan exhibited substantial growth in prescription rates $(2.4 \%$ in 2015 and $13.8 \%$ in 2016). In most of the clinical departments, especially emergency and critical care medicine, neurology, cardiovascular surgery and nephrology, the number of patients prescribed PPIs was up to 3 times higher in the second half of the period (2013-2016) than in the first half of the period (2009-2012). Patients received PPIs concomitantly with NSAIDs and antithrombotic agents has been increased.

Conclusions The use of PPIs, as well as the approved indications for PPIs, has increased substantially in hospitalised patients. The prevalence of PPI prescription by doctors other than gastroenterologists also increased.

\section{IDDF2018-ABS-0061 RISK OF TUBERCULOSIS IN INFLAMMATORY BOWEL DISEASE AND OTHER IMMUNE- MEDIATED DISEASES ON BIOLOGICAL THERAPY: A POPULATION-BASED STUDY IN HONG KONG}

${ }^{1}$ Xing Wang*, ${ }^{2}$ Sunny Wong, ${ }^{2}$ Xian-Song Wang, ${ }^{2}$ Whitney Tang, ${ }^{1}$ Bin Wu, ${ }^{2}$ Justin Wu, ${ }^{2}$ Francis Chan, ${ }^{2}$ Joseph Sung, ${ }^{2}$ Siew Ng. ${ }^{1}$ Department of Gastroenterology, The Third Affiliated Hospital of Sun Yat-sen University, China; ${ }^{2}$ Department of Medicine and Therapeutics, Institute of Digestive Diseases, The Chinese University of Hong Kong, Hong Kong

\subsection{6/gutjnl-2018-IDDFabstracts.93}

Background Biological therapies are increasingly used to treat inflammatory bowel disease (IBD) and other immune-mediated diseases. However, real-world epidemiological data on the risk of tuberculosis (TB) in these patients are scarce. We investigated the incidence of $\mathrm{TB}$ in patients with immune-mediated diseases in a population-based setting and stratified the risk of TB among different biologics.

Methods Data on patient demographics, disease diagnosis, types of biologics and TB infections were collected from a territory-wide computerised database of patient records managed by the Hong Kong Hospital Authority. We calculated the incidence rates (IRs) of TB infections in subjects treated with different biologics between 2006 and 2015, and reported 